\title{
THE DUV-FEL DEVELOPMENT PROGRAM
}

\author{
L.-H. $\mathrm{Yu}^{\dagger 1}$, L. F. DiMauro ${ }^{1}$, A. Doyuran ${ }^{1}$, W. Graves ${ }^{1}$, E. Johnson ${ }^{1}$, S. Krinsky ${ }^{1}$, \\ S. Mikhailov ${ }^{2}$, G. Rakowsky ${ }^{1}$, J. Skaritka ${ }^{1}$, T. Shaftan ${ }^{1}$, B. Sheehy ${ }^{1}$, J.H. Wu ${ }^{1}$ \\ ${ }^{1}$ BNL, Upton, NY 11973, USA \\ ${ }^{2}$ Duke University, Durham, NC 27708, USA
}

\begin{abstract}
We discuss the design and output radiation parameters for the Deep Ultra-violet Free Electron Laser at BNL, which will generate coherent output down to $100 \mathrm{~nm}$ using High Gain Harmonic Generation. The result of FEL calculation and the status of the experiment are presented.
\end{abstract}

\section{INTRODUCTION}

At BNL we are constructing a Deep UVFEL using the high-gain harmonic generation (HGHG) scheme [1] as an intermediate step toward developing a method to generate longitudinally coherent X-rays. In this scheme a coherent seed at a wavelength that is a subharmonic of the desired output radiation interacts with the electron beam in an energy-modulating section. This energy modulation is then converted into spatial bunching while traversing a dispersive section. In the second undulator (the radiator), which is tuned to a higher harmonic of the seed radiation, the microbunched electron beam first emits coherent radiation and then amplifies it exponentially until saturation is achieved. Compared with the SASE scheme, HGHG has a number of advantages, such as nearly Fourier transform limited narrow bandwidth, stable central wavelength, controlled short pulse length down to $10 \mathrm{fs}$, and reduced intensity fluctuation. The HGHG properties have been investigated both theoretically and experimentally in the past several years. Recent results of IR HGHG experiments show good agreement with theoretical expectations [2].

The next step in the development of the HGHG short-wavelength FEL is the DUVEL at BNL. We plan four stages of experiments to gradually generate shorter and shorter wavelength. Starting from a first step of SASE at 400nm using NISUS undulator, the second stage uses the HGHG process to generate 400 $\mathrm{nm}$ from $800 \mathrm{~nm}$. The third stage converts $400 \mathrm{~nm}$ to $200 \mathrm{~nm}$. And at the final stage, with an energy upgrade from $200 \mathrm{MeV}$ to $290 \mathrm{MeV}$, we generate $100 \mathrm{~nm}$ from a $300 \mathrm{~nm}$ seed. The parameters of the electron beam and HGHG radiation for different stages are listed in Table 1. The parameters of the first SASE stage are written in the same column as the second HGHG stage except without the seed laser input. For a relatively conservative parameters, the NISUS undulator has only 10 gain length, not sufficient for SASE saturation, but the simulation shows that it is enough for the HGHG to saturate.

Presently, the system is nearly ready for the first (SASE) stage at $400 \mathrm{~nm}$. In this paper we will mainly discuss the first and second stage aiming to achieve $400 \mathrm{~nm}$ SASE and HGHG lasing.

Table 1. HGHG and electron beam parameters.

\begin{tabular}{|l|l|l|l|}
\hline Parameter \Stage & I, II & III & IV \\
\hline Seed Laser Wavelength, nm & 800 & 400 & 300 \\
\hline Seed Power, MW & 20 & 60 & 90 \\
\hline $\begin{array}{l}\text { FEL output Wavelength, } \\
\text { nm }\end{array}$ & 400 & 200 & 100 \\
\hline FEL output Power, MW & 85 & 180 & 130 \\
\hline Radiator Gain Length, m & 1.05 & 1.12 & 1.14 \\
\hline $\begin{array}{l}\text { Electron beam Energy, } \\
\text { MeV }\end{array}$ & 145 & 210 & 290 \\
\hline $\begin{array}{l}\text { Normalized Emittance, } \pi \\
\text { mm-mrad }\end{array}$ & 5 & 4 & 3 \\
\hline Peak Current, A & 300 & 500 & 1000 \\
\hline Energy spread, $\%$ & 0.15 & 0.15 & 0.15 \\
\hline
\end{tabular}

\section{THE FEL PREPARATION}

The FEL magnetic system consists of the Mini undulator (the modulator), the dispersive section and the NISUS wiggler [3]. Optical system for FEL includes axicon mirror [4] to couple the laser light with the electron beam in the Mini undulator. The harmonic of the same Titanium-Sapphire laser will be used as a seed in the HGHG experiment.

The parameters of the NISUS are listed in Table 2. The wiggler contains 16 segments with 32 poles in each. Six central poles in each segment are canted to introduce the horizontal focusing. Every section includes a "four wire" system to produce additional dipole or quadrupole field to correct beam trajectory and sizes. There are 16 pop-in monitors with YAG screens and OTR mirrors for electron beam and radiation diagnostics. Ten of the 16 OTR ports can be used, five of them will be used for FEL radiation measurements, the other five for OTR measurement will have shadow shields to block the spontaneous 
radiation. The optical spectrometer is installed at the end of the wiggler for the spectrum measurements.

Table 2. The parameters of NISUS wiggler.

\begin{tabular}{|l|l|l|l|}
\hline Period, cm & 3.89 & Wiggler length, $\mathrm{m}$ & 10 \\
\hline Gap, $\mathrm{cm}$ & 2.06 & Field, T & 0.31 \\
\hline
\end{tabular}

One of the important issues in high-gain FEL is the straightness of the electron beam trajectory inside the wiggler. For the parameters listed above (first stage) the deviations of the trajectory from the magnetic axis in the NISUS should not exceed $60 \mu \mathrm{m}$ (RMS). To establish the required accuracy we have developed several methods.

For preliminary alignment we are planning to use He-Ne laser beam, introduced by the seed laser mirror upstream of the NISUS, which will give us a reference to measure the deviations of electron beam trajectory in the monitor locations. The accuracy of this method is mostly determined by the quality of the laser profile image in the monitors. A preliminary test showed that high quality YAG crystal is critical to achieve the required precision. Another important issue is the stability of the laser spot on the monitors. Small vibrations of the laser and matching lenses support can cause the big variations of the centroid position on the monitors due to long laser beam path (the distance between the laser and the last monitor is $20 \mathrm{~m}$ ). In order to overcome this problem we use the "small" feedback system. The laser beam is splitted into two beams, first is used for the alignment and second is the auxiliary beam for the stabilization of the first one. The centroid position of the second beam is recorded by Hamamatsy Tetra-lateral 2-dimensional Position Sensitive Detectors C4674. Feedback system corrects the displacements of the beam centroid on the PSD's generating the current which is proportional to displacement for adjustable support of the laser mirror.

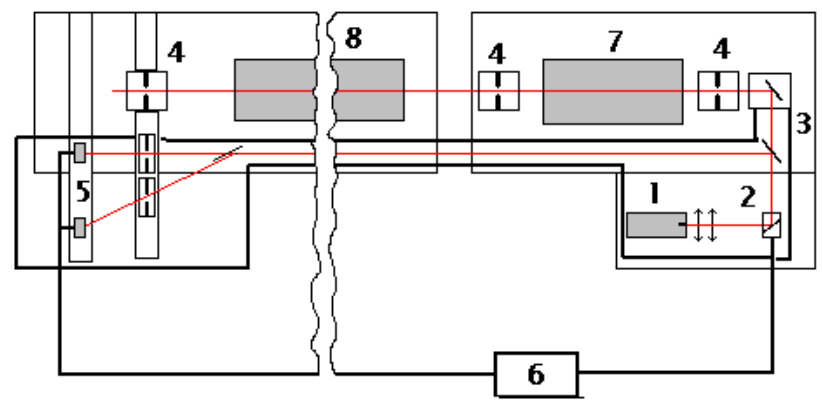

Fig.1 The layout of Alignment Laser Feedback system. 1 - laser, 2 - mirror with adjustable support, 3 - beam splitters, 4 - in-vacuum irises for laser alignment, 5 position sensitive detectors, 7 - Mini-undulator, 8 NISUS wiggler.

For more accurate alignment we shall use the electron beam-based methods. In one of the methods, the position of the electron beam centroid is measured with respect to the "four wire" focusing system. The procedure of alignment is to align the trajectory with the "four wire" magnetic axis. Since the wires are fixed to the vacuum chamber, we are going to control the vacuum chamber alignment with respect to NISUS magnetic axis, using $10 \mathrm{~m}$ long optical rail (deviations from the straight line are less than $6 \mu \mathrm{m}$ for the rail, even though the alignment with respect to the NISUS axis can only be good to $100 \mu \mathrm{m}$ ).

In order to estimate the accuracy of these methods we developed a model of the NISUS wiggler, which includes the fields of canted poles and "four wire" system. It was shown that if there is a $100 \mu \mathrm{m}$ displacement error, the variation of the wire current by \pm 50 ampere (the limit of the power supply) causes more than $\pm 100 \mu \mathrm{m}$ beam movement.

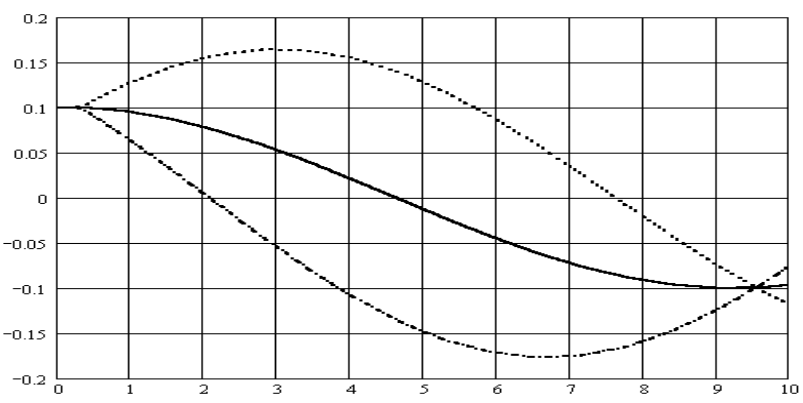

Fig. 5 Vertical trajectories in the NISUS wiggler for $100 \mu \mathrm{m}$ initial displacement. Solid line corresponds to disturbed trajectory with four wire system OFF. Dashed/dotted lines - four-wire system is ON (+50A) / $(-50 \mathrm{~A})$. Vertical scale is in $\mathrm{mm}$, horizontal axis is in $\mathrm{m}$.

Another method is based on the measurements of the trajectories for different energies. In this case the trajectory deviations from the NISUS magnetic axis are inversely proportional to the energy of electron beam. The differences in the centroid positions on every monitor for several energies can be used to determine the deviations of trajectory from the axis.

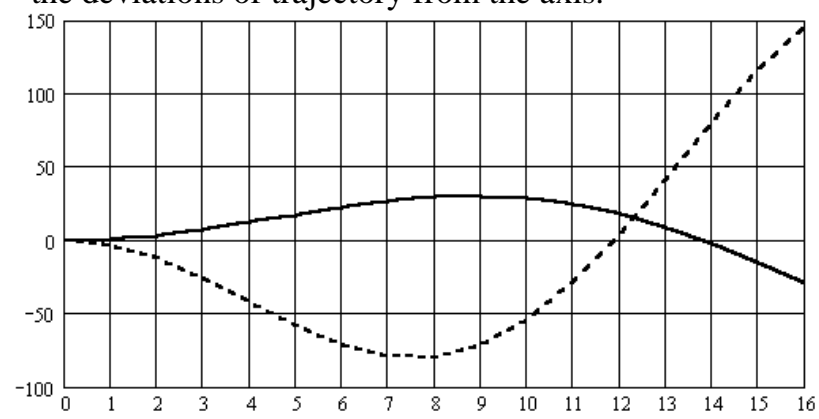

Fig. 6 The differences between disturbed trajectories with different energies (100 and $140 \mathrm{MeV}$ ) of electron beam for $100 \mu \mathrm{m}$ initial offset on $\mathrm{X}$ and 30 urad on Y. Vertical scale is in $\mu \mathrm{m}$, horizontal axis is the monitor number. 


\section{THE COMMISSIONING OF THE SDL ACCELERATOR}

The system consists of the photo-cathode RF gun, a Titanium-Sapphire laser, four SLAC-type linac sections, a four-magnet compressor, electron energy spectrometer and matching optics. The $4.3 \mathrm{MeV}$ electron beam with pulse length of 2 ps (RMS) leaves the gun, accelerates up to $60 \mathrm{MeV}$ with an energy chirp in two linac sections and is compressed in the magnetic chicane down to 0.3 ps. Final acceleration up to $140 \mathrm{MeV}$ is performed in the last two linac sections. (During the commissioning stage, after passing through the spectrometer, the beam is bent to the beam dump.)

In order to establish an accurate beamline optics model we did a set of measurements of electron beam sizes at different monitors. Fig.2 shows a good agreement between MAD program calculations and measured values for given quadrupole and accelerator tank settings. This information is important for the electron beam transport through the NISUS wiggler.
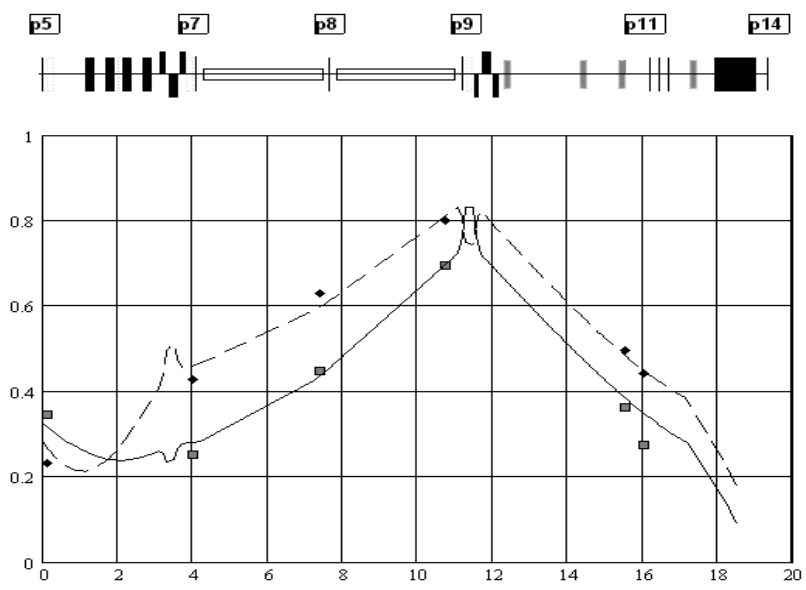

Fig. 2 The comparison between measured and calculated beam sizes. Vertical scale is in $\mathrm{mm}$, horizontal one in $\mathrm{m}$.

The compression was the next important step in the commissioning of the accelerator. We used the dispersion in the dipole after the last linac tank as a spectrometer to measure both energy spread and bunch length. The monitor after the dipole allowed us to measure energy spread up to $1.5 \%$ FWHM with accuracy $0.001 \%$. We had obtained an energy spread of uncompressed bunch as small as $0.01 \%$ for a 250 pC, 4 ps (FWHM) pulse with $4 \pi \mathrm{mm}$-mrad transverse emittances. For the bunch length determination we applied zero-phasing technique [5]. Using the second linac tank to make the energy chirp in the beam we compressed the beam in the chicane, then removed residual chirp in the third tank and measured final energy spread. The fourth linac tank was used to put a linear chirp in the beam and, furthermore, measure the bunch length, which is proportional to the energy spread of the chirped bunch. In order to calibrate maximum energy gain in each tank we measured the dependence of the final beam energy versus tank phase detuning. Fig. 3 shows the dependence of peak current versus time for the compressed electron beam. There is strong evidence that the sharp modulation of peak current is due to laser time structure. We have found that a small tilt of the doubling crystal in the Ti:Sa laser system leads to a dramatic redistribution of the modulation peaks in the beam peak current. This problem may cause degradation of the FEL gain and it is under study now.

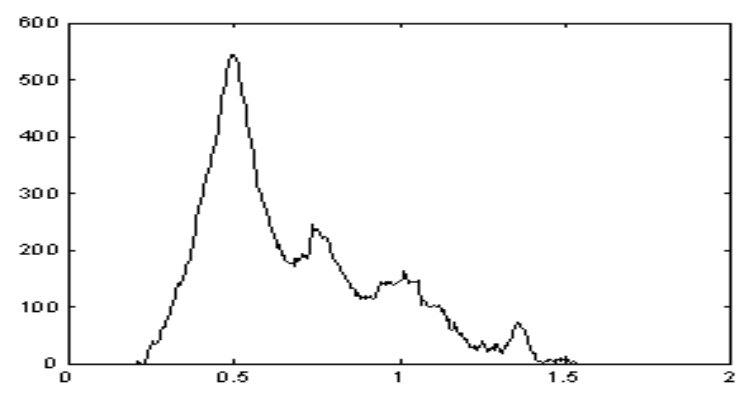

Fig 3. Peak current versus time for the compressed bunch. Bunchlength is equal to $0.52 \mathrm{ps}$ (FWHM).

\section{CONCLUSION}

Recent results of SDL commissioning demonstrate that beam parameters are nearly ready for the generation of SASE and HGHG at $400 \mathrm{~nm}$. Electron beam compression has been done without significant emittance degradation. The diagnostics system for FEL radiation is developed and tested. A model of the NISUS wiggler has been investigated and trajectory control programs have been developed.

\section{REFERENCES}

[1] I. Ben-Zvi, L. F. Di Mauro, S. Krinsky, M.White, L. H. $\mathrm{Yu}$, "Proposed UV-FEL User Facility at BNL", 11'th International Free Electron Laser Conference, Nucl. Instru. Meth., A304, 181 (1991); L. H. Yu, "Generation of Intense UV Radiation by Subharmonically Seeded Single Pass FEL", Phys. Rev. A, 44, 5178 (1991)

[2] L.-H. $\mathrm{Yu}^{\dagger}$, et al., "High-gain harmonic generation free electron laser", Science, 289 (2000)

[3] D.C. Quimby et al, "Development of a 10-meter Wedgedpole undulator," NIM A 285 (1989), pp.281-289.

[4] I.V. Pogorelsky and W.D. Kimura, "Waveguiding by axicon focused laser beams," Adv. Accel. Concepts 1994, pp.419-428

[5] K.N. Ricci, E.R. Crosson, T.I. Smith, "Direct measurement of electron bunch shapes and coherent undulator radiation produced by 100 femtosecond structure," NIM A 445 (2000), pp. 333-337

[6] C. Adolfsen et al., "Beam based alignment technique for the SLC linac,” PAC'89, Chicago, March 1989.

[7] K.Kubo and T.O. Raubenheimer, "Beam based alignment technique for correction of accelerator structure misalignments," LINAC'94, Tsukuba, August 1994. 